\title{
Bionic dentist - Is this the future of dentistry?
}

\author{
Md. NazmulHasan, ${ }^{\mathbf{a}}$ Syed M. S. Islam. ${ }^{\mathbf{b}}$
}

Within the last 25years, the development of digital technology has greatly changed the practice pattern of dentistry. Incorporation of such technology is now becoming an integral part of contemporary dentistry that has largely modified the traditional diagnostic technique,disease interpretations, management protocol, treatment outcome, record keeping even the teaching learning secession in the dental school. Even the symbolic representation of dentistry with a dental mirror and a periodontal probe are becoming rare in use and being replaced by digital intraoral camera and digital pulp tester. Various form of intra oral camera is now a routine record keeping devices in our dental offices. This ensures quick better diagnosis with better light eliminations, better patient's demonstrations. To identify affected teeth inside the mouth using digital pulp tester gives better diagnosis compare to traditional palpation and percussion technique. In the same way intraoral and extra-oral radiographic diagnostic technique has improved by digital radioviosiography (RVG),Cone beam computed tomography (CBCT) that ensure a much better perfect diagnosis compare to the conventional one. ${ }^{2}$ In last year's (October'2012) annual sessions of American Dental Associations (ADA) at San Francisco, with the motto "the future of dentistry meets here" the main focused was how to successfully integrate digital technologies into a dental practice, and several key opinion leaders shared their thoughts on specific products and services and why now is an optimum time to consider "going digital." That was the most "hot topic" in panel discussions. For 15 years it was cosmetics, before that it was implants, and before that it was ${ }_{\text {TMJ. }}{ }^{3}$ Everything is happening faster. Dentistry is going to change more in the next five years than it has in the last 50. The focus on digital radiography, digital impressions, caries detection aids, micromotors, restorative materials, digital denture technology, oral cancer detection, high-tech data backup, light curing, lasers, patient education software, online communication tools, and light systems are already in the tool list of a contemporary digital dentist's. Each area of digital dentistry has advantages in comparison to the conventional device or technique. Yet, some of the advantages may be diminished by the increased cost or technique sensitivity.

Even in the conventional dental education system using of typodont and manikin for clinical purpose has been successfully been replace by robot patients in some institute. ${ }^{4}$ Telemedicine and videoconferencing ${ }^{5}$ also has become more effective way to serve dentistry in community purpose. After successfully using 3D ear and face feature for multibiometric human recognition, ${ }^{6}$ importance of occlusion relationship recording in digital format at dental office has gain more interest for forensic odontologist. So days are very close insurance company might not support those dentists without having proper digital technology. The in-office CAD/CAM systems offer the amazing and distinct advantage of giving full control of the restorative and prostho-dontic process to the dentist. Using this CAD/CAM technology in 
orthodontics invisible braces technique has replace the conventional metal braces. Using non invasiveVELscope light to detect early from of oral cancer has replaced by the conventional surgical collection of biopsy tissue. Digital shade selection of tooth can perform more accurate anterior tooth restoration than conventional one. Occlusion and TMJ analysis and diagnosis of occlusal force equilibrium with software can give more perfect treatment outcome then conventional one.

Digital dentistry is more than just hype. When properly implemented and fully educated, return on investment can be excellent, increased joy in practicing dentistry can be experienced, and better care for your patients can be delivered.The future of dentistry is now, incorporating technology make it a bionic one. Waiting another 10 years to adopt or integrate these new areas of dentistry will leave any onein decades behind. Now everyone is starting to look at the entire thing as a dentist, not just the teeth they have served. So, be digital to be a bionic dentist.

[UpdatDent.Coll.J 2013;3(2):1-2]

a. Md. Nazmul Hasan

Assistant Professor, Department of Orthodontics \&Dentofacial Orthopedics, Update Dental College \& Hospital, Dhaka, Bangladesh,

\section{b. Syed M. S. Islam}

Research Assistant Professor, School of Dentistry, Oral Health Center of Western Australia, The University of Western Australia.

\section{Referances:}

1. Cooley RL, Stilley J, Lubow RM. Evaluations of a digital pulp tester. Oral surgery oral pathology oral medicine. 1984;58(4):437-422.

2. Howerton WB, Mora MA. Advance in the digital imaging: what is new and on the horaizon? The Journal of Americian Dental Associations. 2008;139(suppl 3):20S-24S.

3. Kincade K. DrBicuspid.com[Internet].Tucson(A Z): Kathy Kincade; 2012- Digital dentistry- The future is now;2012 October 29[cited 2013 September $15]$. Available from:http://www.drbicuspid.com/

4. Tanzawa T, Futaki K, Tani C, Hasegawa T, Yamamoto M, Miyazaki T, Maki K. Introduction of a robot patients into dental education. Europian Journal of Dental Education. 2012;16(1):195199.

5. Cook J, Austen G, Stephens C. Videoconferancing: what are the benefits for dental practice? British Dental Journal. 2000;188: 67-70.

6. Islam SMS, Rowan D, Bennamoun M, Owens RA, Mian AS. Multibiometric human recognition using 3D ear and face features. Pattern Recognition. 2013;46(3):613-627. 